\title{
O USO DA MECANOTERAPIA NA PREVENÇÃO DE QUEDAS EM IDOSOS
}

\author{
THE USE OF MECHANOTHERAPY IN THE PREVENTION OF FALLS IN THE \\ ELDERLY
}

\author{
Lauro Mendonça Guimarães ${ }^{1}$ \\ Natanne Maria Da Silva Vieira Borges ${ }^{2}$ \\ Fernando Duarte $\mathrm{Cabral}^{3}$
}

RESUMO: O presente artigo objetivou analisar os benefícios da mecanoterapia relacionado a prevenção de quedas em idosos, visando identificar que forma a mecanoterapia pode contribuir na prevenção de quedas em idosos. Uma vez que, a mecanoterapia é uma técnica que visa melhorar o fortalecimento e resistência muscular, mediante o uso de dispositivos mecânicos fisioterapêuticos, para a melhora na qualidade de vida e prevenindo os riscos de quedas e lesões. A abordagem metodologia ocorreu na revisão bibliográfica, sendo uma pesquisa qualitativa e exploratória, buscou-se nas bases de dados virtuais como, Google acadêmico, Scielo, Hearth Sciences e no acervo da biblioteca universitária, usando o descritor: mecanoterapia, fisioterapia, quedas e idosos. Observou-se nas literaturas uma diversidade significativa de aparelho mecânicos para a utilização de profissionais ao desenvolverem a mecanoterapia, que estas são ferramentas importantes utilizadas em seu cotidiano clínico, mas a eficácia no resultado terá maior probabilidade de ser alcançada se profissional fisioterapeuta estiver sempre em constante lapidação de seus saberes e técnicas, sendo indispensável nesta ação preventiva.

Palavras-Chave: Gerontologia. Distúrbios do envelhecimento. Fisioterapeuta.

ABSTRACT: This article aimed to analyze the benefits of mechanotherapy related to the prevention of falls in the elderly, in order to identify how mechanotherapy can contribute to the prevention of falls in the elderly. Since, mechanotherapy is a technique that aims to improve muscle strength and endurance, through the use of mechanical physiotherapeutic devices, to improve the quality of life and prevent the risks of falls and injuries. The methodology approach occurred in the bibliographic review, being a qualitative and exploratory research, it was searched in virtual databases such as Google academic, Scielo, Hearth Sciences and in the university library collection, using the descriptor: mechanotherapy, physiotherapy, falls and the elderly. It was observed in the literature a significant diversity of mechanical devices for the use of professionals when developing mechanotherapy, that these are important tools used in their clinical routine, but the effectiveness in the result will be more likely to be achieved if a physical therapist is always in constant refining their knowledge and techniques, being indispensable in this preventive action.

Keywords: Gerontology. Aging disorders.Physiotherapist.

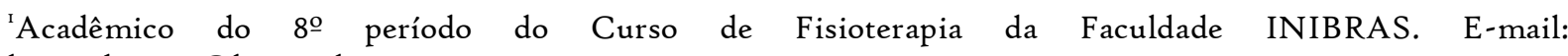
lauro_danone@hotmail.com.

${ }^{2}$ Professora do Curso de Fisioterapia da Faculdade UNIBRAS e orientadora da pesquisa. E-mail: natannevb@gmail.com.

${ }^{3}$ Fernando Duarte Cabral. Professor Curso de Fisioterapia da Faculdade UNIBRAS. E-mail: fernandofisioz@hotmail.com.
} 


\section{INTRODUÇÃO}

O processo envelhecimento vem ganhando destaque devido ao crescimento rápido marcado por um aumento na perspectiva de vida e longevidade. E isso engloba vários aspectos de alterações fisiológicas, físicas, psicológicas, culturais, como a perda da independência funcional e à propensão no desenvolvimento de patologias. Além disso, o mais preocupante nessa situação tem sido correlacionado com a perda de equilíbrio e a diminuição da massa muscular óssea levando a quedas (SOUZA, L., et al., 2017).

O risco de quedas de idosos torna-se uma problemática na saúde pública, visto que, aumenta de maneira significativa com o avançar da idade e está intimamente associado com os distúrbios do envelhecimento resultando em um impacto e custos para a saúde do idoso (CUNHA, 20II).

Os principais fatores que influenciam o risco para quedas são idade avançada, sexo feminino; função neuromuscular prejudicada; ambiente físico inadequado e incapacidade funcional. Em contrapartida, uma intervenção satisfatória nesse caso, é a prática de exercícios físicos que visa minimizar as implicações quanto às quedas (SOUZA, L., et al. 2017).

O Fisioterapeuta é o profissional apto para estar realizando os Exercícios terapêuticos nos idosos. E uma intervenção bastante indicada, é a mecanoterapia. Essa terapia é caracterizada através da mecanoterapia por atividades com auxílios de aparelhos que propõe estimular e recuperar e prevenir possíveis futuras quedas. Deve-se avaliar o indivíduo como um todo e de forma individualizada, planejando um programa de exercícios voltado a necessidade e a capacidade de realizá-los (SOUZA, I., et al., 2019).

É importante ressaltar que, a presente pesquisa primou em fornecer, argumento com maior clareza, sobre os malefícios das quedas pelos idosos, podendo contribuir para o desenvolvimento de ações preventivas no sentido de conscientizar o idoso e a família. As causas são multifatoriais exigem conhecimento e adesão a ações multidisciplinares, a fim de prover identificação dos idosos com risco e estabelecer medidas de prevenção efetivas e eficazes com o intuito de diminuir os danos relacionados a esse evento.

Com o objetivo de analisar os benefícios da mecanoterapia relacionado a prevenção de quedas em idosos, esta pesquisa teve como área de concentração a Gerontologia, visando identificar de que forma a mecanoterapia pode contribuir na prevenção de quedas 
em idosos. Uma vez que, a mecanoterapia é uma técnica que visa melhorar o fortalecimento e resistência muscular, mediante o uso de dispositivos mecânicos fisioterapêuticos, promovendo, portanto, melhora na qualidade de vida e prevenindo os riscos de quedas e lesões.

No que tange a pesquisa e busca de mais conhecimentos para o uso dos dispositivos mecânicos, respalda-se na Resolução CNE/CES 4 , de 19 de fevereiro de 2002, que destaca:

Art. 6o Os conteúdos essenciais para o Curso de Graduação em Fisioterapia devem:

III - Conhecimentos Biotecnológicos - abrange conhecimentos que favorecem o acompanhamento dos avanços biotecnológicos utilizados nas ações fisioterapêuticas que permitam incorporar as inovações tecnológicas inerentes a pesquisa e a prática clínica fisioterapêutica; $e$

Ao buscar descrever sobre o envelhecimento e apontar as principais causas das consequências associadas à queda, fez-se necessário conceituar a mecanoterapia e enfatizar sua importância detalhando ainda, as ferramentas utilizadas nessa técnica dando destaque ao papel do fisioterapeuta frente à assistência ao idoso. Entende-se que os conhecimentos biotecnológicos propostos, a fisioterapia pode levar a caminhos mais eficazes.

\section{METODOLOGIA}

Trata-se de uma abordagem de revisão bibliográfica e exploratória que, segundo Gil (2010) pode-se definir como um procedimento racional, sistemático e reflexivo baseado em métodos específicos permitindo ao pesquisador informações variadas sobre o assunto escolhido, além disso, fornecem subsídios para análise e discussão dos dados ou informações.

Após a definição do tema buscou-se nas bases de dados virtuais como, Google acadêmico, Scielo, Hearth Sciences e no acervo da biblioteca universitária usando o descritor: mecanoterapia, fisioterapia, quedas e idosos. Autores já conhecedores do assunto e estudos realizados para fundamentos desta pesquisa.

Para compor este estudo, utilizou-se critérios com base na seleção de artigos classificados mediante a leitura dos títulos e dos resumos, segundo o tema abordado, artigos obtidos na íntegra, redigidos em português, produzidos nos últimos io anos e excluídos os materiais científicos que não atenderam os objetivos do estudo. 


\section{REFERENCIAL TEÓRICO}

O envelhecimento cresce vertiginosamente no país, em decorrência das mudanças ocorridas nas taxas de natalidade e mortalidade favorecendo o aumento na expectativa de vida. É notório que o país envelhece a passos largos. As alterações na estrutura populacional são claras e irreversíveis. Estima- se que até o ano de 2025 aproximadamente 32 milhões de idosos de brasileiros serão idosos. Com vistas a isto o Brasil ocupará o sexto lugar entre os países com mais idosos no mundo (GUIMARÃES; CUSTÓDIO; AZEVEDO FILHO, 2015).

O envelhecimento é um processo natural que compromete progressivamente os aspectos físicos e cognitivos, está relacionado a um estado biológico de declínio das capacidades físicas, relacionado a uma gama de fragilidades comportamentais e psicológicas. É notório que mesmo sendo natural, é um processo complexo de alterações na trajetória de vida das pessoas. Sendo perceptível a redução da massa e força muscular, perda da capacidade de manter o equilíbrio homeostático, a pessoa fica mais sujeito às perdas evolutivas em vários domínios, diminuindo a reserva funcional, ou seja, a uma somatória de eventos como quedas (GASPAROTTO; FALSARELLA; COIMBRA, 2014).

Souza, L. et al. (2017) informa que Organização Mundial da Saúde (OMS) que pessoas idosas detenham uma maior probabilidade de incapacidade funcional e risco de quedas, pois em uma pesquisa realizada com idosos voluntários, notaram encurtamentos nas pernas de até $3 \mathrm{I} \mathrm{cm}$, essa medida é considerada o melhor e mais sensível parâmetro de massa muscular, por ser de grande precisão nesse público. Além disso, essa medida antropométrica indica mudanças na massa livre de gordura que ocorrem com o avanço da idade e com a redução do nível de atividade física.

\section{I.I O RISCO DE QUEDAS DE IDOSOS}

A queda torna-se um ato frequente, e é caracterizada como um acontecimento não intencional como consequência da alteração da posição inicial para um mesmo nível o mais baixo. As causas podem ser multifatoriais como supracitados anteriormente em resultado da incapacidade funcional (GASPAROTTO; FALSARELLA; COIMBRA, 2014). 


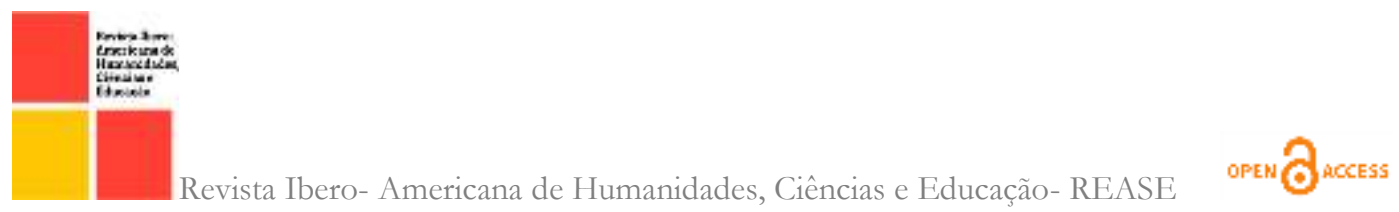

Segundo Rosa, Cappellari e Urbanetto (2019) as quedas estão entre a segunda causa de morte em idosos no mundo de forma acidental ou não intencional, aproximadamente 646.0oo idosos morrem por ano.

As quedas podem estar associadas a fatores extrínsecos, intrínsecos e comportamentais, como diminuição da mobilidade; incontinência urinária, uso de medicamentos, idade avançada, hipotensão postural, pisos desnivelados, altura inadequada da cadeira, cama e do degrau (ROSA; CAPPELLARI; URBANETTO, 2019).

A prevalência de queda tem sido relacionada ao sedentarismo, doenças crônicas, insônia e cochilo. Nesse víeis, estudos apontam que cerca de 60 a 70 \% das quedas acontecem em casa e tem medo de caírem novamente sendo uma possibilidade restringir as atividades, quedas em idosos são mais comuns entre as mulheres, consequentemente levam comprometimento das funções osteomusculares, bem como podem levarem à óbito, (GASPAROTTO; FALSARELLA; COIMBRA, 2014).

A promoção do envelhecimento saudável e a manutenção da máxima capacidade funcional do indivíduo que envelhece, significa uma maior valorização da autonomia e da autodeterminação e a preservação da independência do idoso (GUIMARÃES, et Al. 2004).

Uma forma de prevenção proposto por Gasparotto, Falsarella e Coimbra (2014) é a prática de atividade física como trabalho de força muscular, treino de equilíbrio, propriocepção, preparação de marcha, equilíbrio, hipotensão postural e transferências têm sido atribuídas como fundamental possibilidade de exercício de forte impacto na prevenção de quedas.

Segundo Sá, Bachion e Menezes (2012) os exercícios físicos apresentam três níveis, sendo: Primária voltada para a prevenção do risco de quedas e o desenvolvimento de doenças. A Secundária é um tipo de tardar as alterações provocadas pela patologia existente e a Terciária visa restaurar a funcionalidade de modo a estimular a independência e realização das atividades diárias.

Aborda-se neste trabalho o tratamento Gerontológico em idosos que possibilita o fortalecimento da estrutura muscular e óssea, uma vez que esta área visa e estuda o processo de envelhecimento e o que fazer para se ter uma melhor qualidade de vida ao longo dos anos. Assim, por meio mecanoterapia pretende-se auxiliar na prevenção de quedas, no combate a redução da flexibilidade, no aumento da força e do equilíbrio. 


\section{I.I A ATUAÇÃO DO FISIOTERAPEUTA NA PREVENÇÃO DE QUEDAS EM IDOSOS}

Segundo Machado (2017), no intuito de compreender mais sobre a Fisioterapia a profissão foi regulamentada oficialmente no Brasil via Decreto-Lei no 938 no ano de 1969 e pela Lei Federal no 6.316 em 1975. Uma formação curricular consistente permite ao fisioterapeuta, em sua avaliação ou consulta, a formulação do diagnóstico fisioterapêutico (cinesiológico-funcional). Ressalta-se que, sua conduta é baseada e pautada nos aspectos éticos de sua formação e interação com o paciente, que tem todo um contexto extra lesão envolvido.

A fisioterapia tem por finalidade o estudo e a aplicabilidade deste estudo, no diagnóstico, prevenção e tratamento de disfunções cinéticas funcionais de órgãos e sistemas, sendo uma ciência, em favor do homem no tratar os distúrbios, entre outros da funcionalidade humana. Já o fisioterapeuta busca melhorar o condicionamento físico do paciente mediante a reabilitação motora e muscular. Os benéficos a essa prática são inúmeros como, aumento da flexibilidade, maior independência, melhoria do equilíbrio, aumento da autoestima, autoconfiança e socialização (CUNHA, 20II).

Dentre os profissionais, o fisioterapeuta é responsável por atender esses pacientes como conduta de ministrar, orientar e ensinar restabelecendo as funções e minimizando o desenvolvimento e o agravamento do quadro clínico em decorrências de complicações secundárias a fraturas e/ou lesões por quedas (PIASSAROLI et al., 2012).

A prevenção da queda é de importância inigualável pelos benefícios e na diminuição dos ricos de morbilidade e mortalidade, pelos transtornos ocasionados aos idoso como: hospitalização e o asilamento. "Os programas de prevenção têm a vantagem de, paralelamente, melhorar a saúde como um todo, bem como a qualidade de vida, sendo sua prática especialmente importante para a faixa etária mais idosa" (BUKSMAN et al., 2008, p. I).

Desta forma, é valido ressaltar que o uso de estratégias preventivas ou reparadoras em idosos são indispensáveis. As preventivas também têm que serem endereçadas aos idosos e seus familiares. Quanto aos riscos e danos acometidos, deve-se abranger orientações sobre possíveis quedas, sobretudo os meios para preveni-las. 


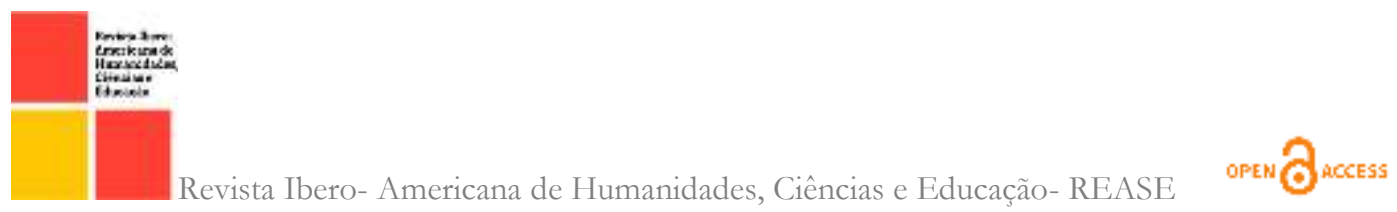

Como ensina, o Ministério da Saúde, (2016). "à elaboração de materiais educativos com subsequente distribuição deles constitui um meio extremamente eficaz e devem ser estimuladas”. Sendo assim, este estudo é um dos meios de orientação e prevenção.

\subsection{HISTÓRIA DA MECANOTERAPIA}

No contexto histórico da mecanoterapia compreende-se sua grande importância no processo de reabilitação, resistência muscular e prevenção de doenças. Ela se define como conjunto de técnicas que utiliza-se de aparelhos mecânicos com finalidade terapêutica. $\mathrm{Na}$ história da fisioterapia já existem relatos de uso de mecanoterapia principalmente em testes musculares na América do Norte. Com a revolução industrial houve o surgimento das primeiras máquinas, ajudando a evoluir esse método de tratamento que utiliza equipamentos mecânicos. (OLIVEITA ET AL, 2019).

O método foi criado pelo médico sueco Gustav Zander, tendo implantando sua metodologia no ano de 1890 , definida por ele como: "o emprego de meios mecânicos para a cura de doenças". Formado no Instituto Karolinka direcionado para tratamento médico através de máquinas especiais no ano de 1890 . Ainda, em 1865 fundou, em Estocolmo, o Medico-mekaniska Institutet (Instituto Médico-Mecânico) para fisioterapia através de máquinas mecânicas, abrindo filiais em Londres e nos Estados Unidos, a partir de então sua metodologia se estendeu ao mundo (OLIVEITA ET AL, 2019).

\subsection{MECANOTERAPIA NO TRATAMENTO DE IDOSOS}

Com vistas neste estudo, concentrou-se esforços para conhecer mais sobre a fisioterapia geriátrica aplicada por meio da mecanoterapia, enfatizando os limites e cuidados necessários através dos procedimentos e métodos adequados para o uso da mecanoterapia.

A fisioterapia gerontológica consiste em uma abordagem integral e humanizada do idoso, enfocando as particularidades do processo de envelhecimento. É precioso valorizar a singularidade do idoso, e todo que envolve seu contexto, assim o fisioterapeuta deve tratar o idoso como um todo, utilizando recursos terapêuticos para prevenção em favor de seu bem-estar, qualidade de vida e de sua saúde.

Como já enfatizado, um dos recursos terapêuticos proposto pelo fisioterapeuta na prevenção das quedas aos idosos é a mecanoterapia, que é uma ferramenta clínica que 
possibilita vantagens interessantes aos pacientes, pois apresenta uma diversidade de exercícios terapêuticos que podem ser aplicados no ambiente ambulatorial e residencial. Ela consiste em movimentos com os auxílios de aparelhos mecânicos, como halteres, molas, elásticos (PIASSAROLI et al., 2012).

O estudo proposto por Bertoldi, Silva e Navega (2013), em pacientes com doença de Parkinson o uso da mecanoterapia com cadeira adutora; mesa flexoextensora teve um resultado satisfatório em busca de aumentar a força muscular, o equilíbrio e a qualidade de vida, minimizando os riscos de quedas que são frequentes nesse pacientes, tendo um percentual de aproximadamente $68 \%$.

Dentre os principais aparelhos utilizados no auxílio da mecanoterapia, encontra-se o cicloergômetro com finalidade da recuperação funcional dos membros inferiores e superiores; prono-supinador auxiliam em patologias que acometem os membros superiores e fortalecem os músculos; rolo de punho e exercitador de dedos apresenta ganhos de amplitude de movimento e fortalecimento dos músculos. Essas atividades podem ser realizadas em clínicas e residências, com atendimento domiciliar, posto que a tecnologia junto com o profissional busca maneiras assertivas e de portabilidade desses equipamentos (SOUZA, I., et al., 2019).

As intervenções quanto aos exercícios e o tratamento mecanoterapêuticos aos idosos proporcionam melhora na capacidade funcional e aptidão física evitando a imobilidade e redução da incidência nos acidentes de quedas. É necessário que o fisioterapeuta avalie o estado geral do idoso, bem como as patologias presentes, o grau de comprometimento, o porte físico, histórico de lesões, nível de mobilidade muscular, as perdas físicas como massa muscular, os exames de sangue, e as orientações dos demais profissionais para planejar o programa de atividades de acordo a funcionalidade $e$ capacidade de cada um, para conseguir chegar ao objetivo proposto (CUNHA, 20II).

Compreende-se ainda, que o auxílio da mecanoterapia na rotina de idosos pode beneficiar em suas Atividades de Vida Diárias (AVDs), pois estudos mostram que alterações no desempenho das AVDs do idoso, pode agravar nos estágios iniciais da demência, e a gravidade do processo demencial foi apontada como um forte preditor para o declínio ainda mais severo de sua rotina ( HILL; BACKMAN; FRATIGLIONI, 20Io). 


\subsection{EQUIPAMENTOS MAIS USADOS PELA MECANOTERAPIA PARA TRATAMENTOS EM IDOSOS}

Os equipamentos mais utilizados podem ser separados em equipamentos para membros superiores e inferiores e aparelhos para tronco, cabeça e pescoço. A finalidade e necessidade dos trabalhos na mecanoterapia, é identificada, prioritariamente, pelas características do tipo de contração muscular adequada pelo fisioterapeuta, atendendo a um determinado propósito, e neste estudo tenciona-se a prevenção de quedas em idosos (BRANDT, Et Al, 2010).

- Aparelhos para membros superiores: mesa de kanavel, finger flex, escada de ombro, polias e bastão;

- Aparelhos para membros inferiores: bicicleta ergométrica, pedalinho para fisioterapia, esteira, mesa de quadríceps ou de bonet, quadro de quadríceps, exercitador de tornozelo e cama elástica;

- Aparelhos para tronco, cabeça e pescoço: tração cervical, tração torácica, tração lombar e outros.

\section{CONSIDERAÇÕES FINAIS}

Foi considerado que através da mecanoterapia é possível viabilizar exercícios para membros superiores, membros inferiores, marcha, equilíbrio, flexibilidade, fortalecimento muscular, resistência muscular e atividades funcionais. Estes exercícios e aquisições são indispensáveis para prevenir a queda em idosos.

A prevenção da queda é indispensável na qualidade e bem-estar dos idoso, concluise que pelos benefícios e na diminuição dos ricos de morbilidade e mortalidade, pelos transtornos ocasionados aos idoso como: hospitalização e o asilamento, são possíveis com o uso da mecanoterapia uma vez que a contração muscular proporcionada no tratamento pode contribuir para fortalecimento, equilíbrio e flexibilidade deste pacientes .

A escolha dos Recursos mecânicos deve sempre ser realizada com muita responsabilidade pelo fisioterapeuta e após uma avaliação fisioterapêutica minuciosa e bem embasada com relação aos objetivos e condutas terapêuticas.

$\mathrm{Na}$ revisão literária foi possível listar os aparelhos para melhorar a mobilidade e demonstrar parâmetros mais utilizados dentro da prática clínica e da literatura 
especializada, além de servir como referencial para posteriores estudos sobre a eficácia/eficiência da mecanoterapia no tratamento fisioterapêutico para idosos.

\section{REFERÊNCIAS}

BERTOLDI, F. C.; SILVA, J. A. M. G.; NAVEGA, F. R. F. Influência do fortalecimento muscular no equilíbrio e qualidade de vida em indivíduos com doença de Parkinson. Fisioter Pesq, v.20, n.2, 2013. Disponível em:< http://www.scielo.br/scielo.php?script=sci_arttext\&pid=SI809-29502013000200004>. Acesso em: 3I mar.2020.

BRANDT, A. C.; Et Al. Um novo recurso de mecanoterapia visando a manutenção de força muscular em membros superiores de pacientes paraplégicos. LILACS. Biblioteca de Ciências da Saúde, digital I5(I):47-54, abr.-set. 2010.

BUKSMAN, S. et al. Quedas em idosos: prevenção. Projeto Diretrizes - Associação Médica Brasileira e Conselho Federal de Medicina, 2008. Disponível em: < http://sbgg.org.br/wp-conte nt/uploads/2014/ro/queda-idosos.pdf $>$. Acesso em: 30 maio 2020 .

CNE. Resolução CNE/CES 4/2002. Diário Oficial da União, Brasília, 4 de março de 2002. Seção I, p. II.

CUNHA, M. G. L. Exercício Físico no contexto da prevenção de quedas em idosos: uma revisão sistemática da literatura. 62p. 20II. Instituto Politécnico de Viseu. Viseu, 20Ir. Disponível:<

https://repositorio.ipv.pt/bitstream/10400.19/1664/I/CUNHA\%20Maria\%20Gra\%(3\%A7 a\%20Lopes\%20-\%20DissMestr\%20-

\%20\%20EXERC\%C3\%8DCIO\%20F\%C3\%8DSICO\%20NO\%20CONTEXTO\%20DA\%20 PREVEN\% $\mathrm{C}_{3} \% 87 \% \mathrm{C}_{3} \% 83 \mathrm{O}$.pdf $>$. Acesso em: 31 mar.2020.

GASPAROTTO, Livia Pimenta Reno; FALSARELlA, Gláucia Regina; COIMBRA, Arlete Maria Valente. As quedas no cenário da velhice: conceitos básicos e atualidades da pesquisa em saúde. Revista Geriatr. Geront, Rio de Janeiro, v.I7, n.I, 20I4. Disponível em:< http://www.scielo.br/pdf/rbgg/vi7nI/r8o9-9823-rbgg-17-oI-oo2or.pdf>. Acesso em: 30 mar.2020

GIL, A. C. Como elaborar projetos de pesquisa. 4.ed. São Paulo. Atlas, 2010.

GUIMARÃES, L. H. C. T. et al. Avaliação da Capacidade Funcional de Idosos em Tratamento Fisioterapêutico. Revista Neurociências 2004. Disponível em:< http://www.revistaneurociencias.com.br/edicoes/2004/RN\%2012\%2003/Pages\%2ofrom\%2 oRN\%2012\%2003-3.pdf $>$. Acesso em: 25 mar. 2020.

GUIMARÃES, A. P. R.; CUSTÓDIO, S. H.; AZEVEDO FILHO, E. R. A contribuição do enfermeiro na recuperação de idosos depressivos. Núcleo Interdisciplinar de Pesquisa. Brasília, 2015 .

Disponível em:< 
http://nippromove.hospedagemdesites.ws/anais_simposio/arquivos_up/documentos/arti gos/o8icb6f3bdeor40oo6ogd236c0299ob9.pdf >. Acesso em: 28 mar.2020.

HILL, R.D.; BACKMAN, L.; FRATIGLIONI, L. Determinants of functional abilities in dementia. J Am Geriatr Soc. 2010;43(10):I-9.

MACHADO, D. A FORMAÇÃO ÉTICA DO FISIOTERAPEUTA». Fisioterapia em Movimento. v. 20, n.3, 2017. ISSN I980-5918. Consultado em I8 de maio de 2020.

MINISTÉRIO DA SAÚDE. Quedas em idosos. Ministério da Saúde. Biblioteca Virtual em Saúde, 2or6. Disponível em: http://bvsms.saude.gov.br/bvs/dicas/I84queda_idosos.html>. Acesso em: 29 mar. 2020.

OLIVEIRA, A.C. B. de et al. MECANOTERAPIA E FACILITAÇÃO NEUROMUSCULAR PROPRIOCEPTIVA: REVISÃO DE LITERATURA. Mostra de Fisioterapia da Unicatólica, [S.1.], v. 3, n. I, feb. 2019. ISSN 2526-4915. Disponível em: $\langle$ http://publicacoesacademicas.unicatolicaquixada.edu.br/index.php/mostrafisioterapia/ar ticle/view/2825>. Acesso em: o6 out. 2020.

PIASSAROLI, C.A.P. et al. Modelos de Reabilitação Fisioterápica em pacientes adultos com sequelas de AVC isquêmico. Rev Neurocien, v.20, n.I, 2012. Disponível em:<http://www.revistaneurociencias.com.br/edicoes/2012/RN200I/revisao\%2020\%200I/6 34\%2orevisao.pdf $>$. Acesso em: mar.2020.

ROSA, V. P. P.; CAPPEllari, F. C. B. D.; URBANETTO, J. S. Análise dos fatores de risco para queda em idosos institucionalizados. Revista Bras. Geriatr. Gerentol, v.22, n.I, 2019. Disponível em:< http://www.scielo.br/scielo.php?script=sci_arttext\&pid=Sı8o99823201900o100208\&lng=pt\&nrm=iso\&tlng=pt $>$. Acesso em: 29 mar.2020.

SÁ, A. C. A. M.; BACHION, M. M.; MENEZES, R. L.. Exercício físico para a prevenção de quedas: ensaio clínico com idosos institucionalizados em Goiânia, Brasil. Ciência\&Saúde, $\quad$ v.I7, $\quad$ n.8, 2012. Disponível em:<

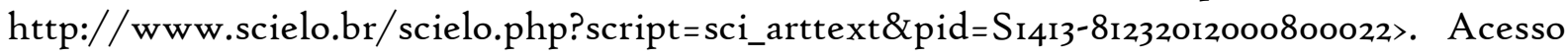
em: 3I mar.2020.

SOUZA, I. M. et al. Inovação em mecanoterapia: implementação de recursos e desenvolvimento de um cicloergômetro sustentável. Rev Cient da Fac Educ e Meio Ambiente, Ariquemes, v.Io, n.I, 2019. Disponível em:< http://www.faema.edu.br/revistas/index.php/Revista-FAEMA/article/view/758>.

Acesso em: 31 mar.2020.

SOUZA, L.H.R. et al. Queda em idosos e fatores de riscos associados. Rev. Aten. Saúde, São Caetano do Sul, v.15, n.54, 2017. Disponível em:< https://webcache.googleusercontent.com/search?q=cache:IwIERCtPMiMJ:https://seer.us cs.edu.br/index.php/revista_ciencias_saude/article/download $/ 4804 / \mathrm{pdf}+\& \mathrm{~cd}=6 \& \mathrm{hl}=\mathrm{pt}-$ $\mathrm{BR} \& \mathrm{ct}=\mathrm{clnk} \& \mathrm{gl}=\mathrm{py}>$. Acesso em: 31 mar.2020. 